\title{
A similarity of quantum phase transition and quench dynamics in the Dicke model beyond the thermodynamic limit
}

\author{
Lituo Shen ${ }^{1}$, Zhicheng Shi ${ }^{1}$, Zhenbiao Yang ${ }^{1}$, Huaizhi Wu ${ }^{1}$, Zhirong Zhong ${ }^{1}$ and Shibiao Zheng ${ }^{1 *}$
}

\section{"Correspondence:}

sbzheng11@163.com

${ }^{1}$ Fujian Key Laboratory of Quantum Information and Quantum Optics, College of Physics and Information Engineering, Fuzhou University, Fuzhou, China

\section{Springer}

\begin{abstract}
We study the quantum phase transition in the Dicke model beyond the thermodynamic limit. With the Kibble-Zurek mechanism and adiabatic dynamics, we find that the residual energy is inversely proportional to the number of qubits, indicating that more qubits can obtain more energies from the oscillator as the number of qubits increases. Finally, we put forward a promising experiment device to realize this system.
\end{abstract}

PACS Codes: $05.30 . \mathrm{Rt} ; 03.75 . \mathrm{Kk} ; 32.80 . \mathrm{Qk}$

Keywords: Quantum phase transition; Quench dynamics; Dicke model; Kibble-Zurek mechanism; Thermodynamic limit

\section{Introduction}

Superradiance is the spontaneous emission of photons from the collective decay of an excited population of atoms. This phenomenon is first predicted in the quantum phase transition (QPT) of the Dicke model [1] and has been experimentally observed in several quantum optical and solid-state systems [2]. The Dicke model describes the interaction between $N$ identical qubits and a single bosonic mode, which exhibits a second-order QPT at weak coupling in the thermodynamic limit $N \rightarrow \infty$ [3]. The superradiant QPT of the Dicke model has been experimentally observed with a Bose-Einstein condensate trapped in an optical cavity [4]. On the other hand, there has been much interest in the realization of QPT of the Dicke model in circuit QED systems due to the theoretical and experimental progresses on the ultrastrong coupling in recent years [5-24]. In thermodynamic limit, the Dicke model is exactly solvable through the Holstein-Primakoff transformation for the angular momentum algebra $[25,26]$.

The universality of equilibrium and nonequilibrium dynamics is important to characterize critical exponents of the QPT [27]. Scaling functions go beyond critical exponents and extend the range where theoretical predictions can agree with experimental datas. Many-body systems can show up highly nontrivial behaviors [28] even for an adiabatic evolution $[29,30]$. For a slow quench dynamics, the adiabaticity breaks down regardless

(c) The Author(s) 2020. This article is licensed under a Creative Commons Attribution 4.0 International License, which permits use, sharing, adaptation, distribution and reproduction in any medium or format, as long as you give appropriate credit to the original author(s) and the source, provide a link to the Creative Commons licence, and indicate if changes were made. The images or other third party material in this article are included in the article's Creative Commons licence, unless indicated otherwise in a credit line to the material. If material is not included in the article's Creative Commons licence and your intended use is not permitted by statutory regulation or exceeds the permitted use, you will need to obtain permission directly from the copyright holder. To view a copy of this licence, visit http://creativecommons.org/licenses/by/4.0/. 
of the quench rate when the system across the critical point, which can be analyzed by the Kibble-Zurek mechanism (KZM) [12].

For realizing the QPT dynamics in experiment [10-13], the major challenge is the condition for thermodynamic limit where the number of the constituent system elements is required to be infinite. Recently, much attention has been paid to the QPT of finitesize many-body systems. However, beyond the thermodynamic limit, the Dicke model is nonintegrable due to the unclosed Hilbert space in a general coupling range [31], so that various approximate methods are proposed to obtain the effect of finite-size correction [32-37], which is demonstrated to be crucial to understand the properties of universality near the critical point of QPT [38-41]. Previous study [32] applied the method of BornOppenheimer approximation to analyze the second-order QPT for the adiabatic Dicke model in the thermodynamic limit. Vidal and Dusuel [33] focused on the finite-size corrections in the Dicke model and determined the analytical scaling exponents at the critical point for various atomic observables. There has been reported [35] that it was able to use extended bosonic coherent states to numerically solve the finite-size Dicke model, but this method can not be directly applied to investigate the QPT due to their lack of closed form.

Based on Schrieffer-Wolff transformation, recent work demonstrated that both the finite-size Jaynes-Cummings lattice system [42] and Rabi model [12] exhibited the superradiant QPT even when the system involved only one qubit and one harmonic oscillator. A natural generalization of the Jaynes-Cummings [42-45] and Rabi [46-60] models is the system involving finite $N$ qubits simultaneously interacting with a common harmonic oscillator, i.e., the Dicke model beyond the thermodynamic limit. Such unitary transformation is very common in the context of spin boson problems which is also known as "polaron transformation". Although several mathematics approaches [32-35] have been presented to analytically treat the finite-size Dicke model, few can be directly applied to treat QPT in the Dicke model beyond the thermodynamic limit.

In this paper we study QPT of the finite-size Dicke model. With the Kibble-Zurek mechanism and adiabatic dynamics, we find that the residual energy is inversely proportional to the number of qubits, indicating that more qubits can "absorb" more energies from the oscillator as the number of qubits increases.

\section{System Hamiltonian}

The Dicke Hamiltonian [1] models the mutual interaction between $N$ identical qubits and a harmonic oscillator, which is described as

$$
\begin{aligned}
& H=H_{0}-V, \\
& H_{0}=w_{0} a^{\dagger} a+\Omega J_{z}, \\
& V=\lambda\left(a^{\dagger}+a\right)\left(J_{+}+J_{-}\right),
\end{aligned}
$$

$w_{0}$ and $\Omega$ are the frequencies of the oscillator and qubit, respectively. $\lambda$ is the qubitoscillator coupling strength. $a^{\dagger}(a)$ is the creation (annihilation) operator of the harmonic oscillator. $J_{z}$ and $J_{ \pm}$are the collective qubit operators and obey the general angular momentum commutation relations

$$
\begin{aligned}
& {\left[J_{z}, J_{ \pm}\right]= \pm J_{ \pm},} \\
& \left.J_{+}, J_{-}\right]=2 J_{z} .
\end{aligned}
$$


The Hilbert subspaces of collective qubit operators and oscillator are denoted by $\Gamma_{Q}=$ $\{|j, m\rangle ; m=-j,-j+1, \ldots, j-1, j\}$ and $\Gamma_{O}=\{|n\rangle ; n=0,1,2, \ldots\}$, respectively, where $j=$ $N / 2$ and $a^{\dagger} a|n\rangle=n|n\rangle$. The parity operator $\Pi=e^{i \pi\left(a^{\dagger} a+J_{z}+j\right)}$ commutes with $H$ and has two eigenvalues \pm 1 which correspond to two noninteracting subspaces, depending on whether the total excitation number is even or odd, respectively. In the thermodynamic limit $N \rightarrow \infty$, in which the number of qubits becomes infinite, the Dicke Hamiltonian has been demonstrated to undergo a second-order QPT [25]. Hereafter, we focus on the nonequilibrium dynamics of the QPT in the Dicke model with a finite number of qubits.

Based on Schrieffer-Wolff transformation, we find an anti-Hermitian operator to map the finite-size Dicke Hamiltonian into an oscillator Hamiltonian within the collectivequbit subspace. By means of Born-Oppenheimer approximation, we analytically derive the eigenenergy and eigenstate of the normal and superradiant phases when the ratio of the qubit transition frequency to the oscillator frequency approaches infinity, and demonstrate that the ground state undergoes a second-order quantum phase transition at a new critical point, where the effective qubit-oscillator coupling strength is quadratically enhanced by the number of qubits. For the finite-frequency scaling, we derive an analytical leading-order correction by using the variational method, as proved in the Appendices A to E.

\section{Quench dynamics}

To investigate the nonequilibrium dynamics of the QPT in the $N$-qubit Dicke model, we focus on a slow quench dynamics based on the adiabatic perturbation theory and KibbleZurek mechanism [61, 62]. Similar to Ref. [12], we consider the control parameter $g$ to change linearly with time, i.e., $\lambda(t)=\sqrt{w_{0} \Omega} \lambda_{f} t /\left(2 \sqrt{N} \tau_{q}\right)$, where $\lambda_{f}$ is the final coupling strength $\left(\lambda_{f} \leq 1\right)$ and $\tau_{q}$ is the quench time. For simplicity, we discuss the situation $g(t) \leq 1$ in the following.

Suppose that the instantaneous eigenstates of the time-dependent Hamiltonian $H_{n p}(t)$ are $\left|r_{n p}(t), n\right\rangle=P\left[r_{n p}(t)\right]|n\rangle$ and the corresponding instantaneous eigenenergies are $\epsilon_{n}(t)=$ $n \epsilon_{n p}(t)$. In terms of the time-dependent wave function $|\Psi(t)\rangle=\sum_{n} \beta_{n}(t) e^{-i \Theta_{n}(t)}\left|r_{n p}(t), n\right\rangle$, where $\Theta_{n}(t)=\int_{0}^{t} \epsilon_{n}\left(t^{\prime}\right) d t^{\prime}$, the Schrödinger equation is equivalent to the following form

$$
\dot{\beta}_{n}(t)=-\sum_{m} \beta_{m}(t)\left\langle r_{n p}(t), n\left|\partial_{t}\right| r_{n p}(t), m\right) e^{i\left[\Theta_{n}(t)-\Theta_{m}(t)\right]},
$$

and the general solution of Eq. (5) is written as

$$
\begin{aligned}
\beta_{n}(\lambda)= & -\sum_{m} \int_{0}^{\lambda} d \lambda^{\prime} \beta_{m}\left(\lambda^{\prime}\right)\left\langle r_{n p}\left(\lambda^{\prime}\right), n\left|\partial_{\lambda^{\prime}}\right| r_{n p}\left(\lambda^{\prime}\right), m\right\rangle \\
& \times e^{i\left[\Theta_{n}\left(\lambda^{\prime}\right)-\Theta_{m}\left(\lambda^{\prime}\right)\right]} .
\end{aligned}
$$

When the system is initially in its ground state with $\beta_{0}(0)=1$, for a slow quench $\dot{g} \ll 1$ and by keeping its leading order, Eq. (6) approximates

$$
\beta_{n}(\lambda) \simeq-\int_{0}^{\lambda}\left\langle r_{n p}\left(\lambda^{\prime}\right), n\left|\partial_{\lambda^{\prime}}\right| r_{n p}\left(\lambda^{\prime}\right), 0\right\rangle e^{i\left[\Theta_{n}\left(\lambda^{\prime}\right)-\Theta_{0}\left(\lambda^{\prime}\right)\right]} .
$$

To deal with the phase factor, we use the approximate evaluation equation of a fast oscillating integral $\int_{x_{1}}^{x_{2}} f(x) e^{i a h(x)} d x=\left.\frac{f(x)}{i a h^{\prime}(x)} e^{i a h(x)}\right|_{x_{1}} ^{x_{2}}+O\left(a^{-2}\right)$ and obtain the equivalent solution 
of Eq. (7) as

$$
\left.\beta_{n}(\lambda) \simeq i \dot{\lambda} \frac{\left\langle r_{n p}(\lambda), n\left|\partial_{\lambda}\right| r_{n p}(\lambda), 0\right\rangle}{\epsilon_{n}(\lambda)-\epsilon_{0}(\lambda)} e^{i\left[\Theta_{n}(\lambda)-\Theta_{0}(\lambda)\right]}\right|_{0} ^{\lambda}
$$

In the normal phase, $r_{n p}(\lambda)=-\frac{1}{4} \ln \left[1-4 N \lambda^{2} /\left(w_{0} \Omega\right)\right]$ and

$$
\begin{aligned}
\left\langle r_{n p}(\lambda), n\left|\partial_{\lambda}\right| r_{n p}(\lambda), 0\right\rangle & =\frac{1}{2} \frac{\partial r_{n p}(\lambda)}{\partial \lambda}\left\langle n\left|\left(a^{\dagger 2}-a^{2}\right)\right| 0\right\rangle \\
& =\frac{\sqrt{2} N \lambda \delta_{n, 2}}{w_{0} \Omega-4 N \lambda^{2}}
\end{aligned}
$$

where the symbol $\delta$ represents the delta function. Therefore, when the higher-order term $O\left(\dot{g}^{2}\right)$ is omitted, the only nonzero solution in Eq. (8) is $\beta_{2}$, given by

$$
\beta_{2}(\lambda) \simeq \frac{\sqrt{2} i \sqrt{N} \dot{\lambda} \lambda}{2 w_{0}^{2} \Omega\left(1-\frac{4 N \lambda^{2}}{w_{0} \Omega}\right)^{z v+1}} e^{i\left[\Theta_{2}(\lambda)-\Theta_{0}(\lambda)\right]} .
$$

According to the adiabatic perturbation theory [63], the final residual energy becomes

$$
\begin{aligned}
E_{r} & =\sum_{n>0} \epsilon_{n}\left(\frac{\sqrt{w_{0} \Omega} \lambda_{f}}{2 \sqrt{N}}\right)\left|\beta_{n}\left(\frac{\sqrt{w_{0} \Omega} \lambda_{f}}{2 \sqrt{N}}\right)\right|^{2} \\
& \simeq \epsilon_{2}\left(\frac{\sqrt{w_{0} \Omega} \lambda_{f}}{2 \sqrt{N}}\right)\left|\beta_{2}\left(\frac{\sqrt{w_{0} \Omega} \lambda_{f}}{2 \sqrt{N}}\right)\right|^{2} \\
& =\tau_{q}^{-2} \frac{w_{0} \lambda_{f}^{4}}{16 N\left(1-\lambda_{f}^{2}\right)^{z v+2}} \\
& \propto \frac{\tau_{q}^{-2}}{N} .
\end{aligned}
$$

Thus, the residual energy $E_{r}$ scales with $\tau_{q}^{-2} / N$ when the quench is far below the critical point $g \ll 1$, which is different from the universal scaling $E_{r} \sim \tau_{q}^{-2}$ in the Rabi model. However, as $\lambda(t)$ approaches the critical point, the relaxation time of the system diverges due to the singularity of the spectral gap, and the scaling relation $E_{r} \sim \tau_{q}^{-2} / N$ does not hold near the critical point. Based on KZM, there exists a time instant $T$ that divides the whole dynamics into two regimes: one is the adiabatic regime below the coupling instant $\lambda(T)$ and the other is the impulsive regime beyond $\lambda(T)$. Since the relation time is calculated through the inverse of the accessible energy-gap equation $\eta(\lambda)=2 \epsilon_{n p}(\lambda)$, the solution of $\lambda(T)$ should satisfy the nonlinear differential equation

$$
\left|\frac{1}{\eta[\lambda(t)]}\right|=\left|\frac{\eta[\lambda(t)]}{\dot{\eta}[\lambda(t)]}\right|
$$

By replacing $\eta(\lambda)=2 w_{0} \sqrt{1-4 N \lambda^{2} /\left(w_{0} \Omega\right)}$ into Eq. (12), we obtain the effective scaling relation between $\lambda(T)$ and $\tau_{q}$ as

$$
\lambda(T)=\frac{\sqrt{w_{0} \Omega}}{2 \sqrt{N}}\left[1-\left(2 \sqrt{2} w_{0} \tau_{q}\right)^{\frac{-1}{z v+1}}\right] .
$$


Figure 1 Residual energy $E_{r}$ versus the quench time $\tau_{q}$ obtained by KZM for different $N$ values: (a) $E_{r} \sim \tau_{q}^{-2} / N$, (b) $E_{r} \sim \tau_{q}^{-1 / 3} / N$. The curves from the top to the bottom correspond to $N=1$ to 10
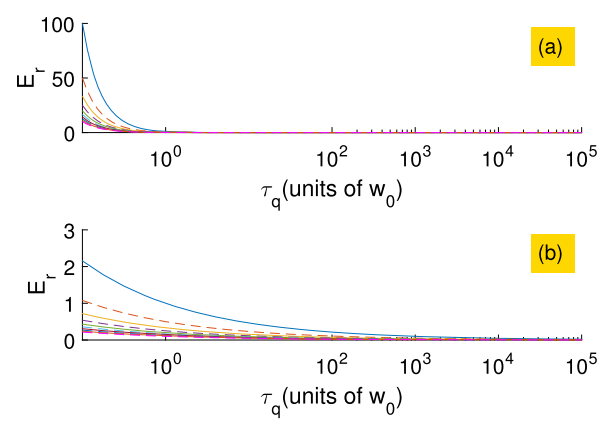

Figure 2 (a) Schematic of N identical NV centers located near a current-carrying nanotube, and there has no direct interaction between arbitrary two driven NV centers. I is the electric current and $B$ is the magnetic field in the nanotube. $d$ is the distance between the nanotube and NV centers, and varies

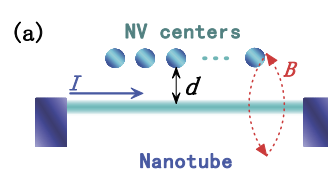

(b)

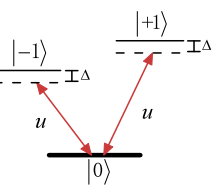
when the nanotube vibrates. The blue-dot line

represents the other NV centers. (b) Level configuration of each driven NV center. $u$ is the driving of external microwave field and $\Delta$ is the symmetric detuning

When the system approaches the critical point, the residual energy becomes

$$
E_{r} \simeq \tau_{q}^{-2} \frac{w_{0}}{16 N}\left(2 \sqrt{2} w_{0} \tau_{q}\right)^{\frac{z v+2}{z v+1}} \propto \frac{\tau_{q}^{-1 / 3}}{N} .
$$

This result shows that when the quench dynamics approaches to the critical point, $E_{r}$ grows inversely proportional to the number of qubits, which is different from the universal scaling $E_{r} \sim \tau_{q}^{-1 / 3}$ in the Rabi model [12]. In Fig. 1, we plot the curves of residual energy $E_{r}$ versus the quench time $\tau_{q}$ obtained by KZM for different $N$ values. The results in Fig. 1(a) and 1(b) show that the residual energy more quickly decrease when the number of qubits increases, indicating the cooperation of the qubits enhances quench effect. The above inverse-proportion scalings between $E_{r}$ and $N$ physically come from the collective enhancement effect among $N$ qubits and this effect vanishes when there is only one single qubit, indicating that more qubits can "absorb" more energies from the oscillator as the number of qubits increases, and the energy exchange between the qubits and the oscillator does not affect the total energy.

\section{Feasible experimental system}

Hybrid quantum device with a nitrogen-vacancy (NV) center in diamond coupled to a current-carrying nanotube has been demonstrated to be well mapped to the standard single-qubit Dicke model [64]. Without loss of generality, we generalize this hybrid quantum device to a more common situation where $N$ identical NV centers collectively coupled to a current-carrying nanotube and there has no direct interaction between arbitrary two driven NV centers when each NV center is far away from the others, as shown in Fig. 2. Each NV center in diamond consists of a substitutional nitrogen atom and an adjacent vacancy, which has one ground state with zero-field splitting between the ground state $|0\rangle$ and excited states $| \pm 1\rangle$. External microwave fields $u$ cause Rabi oscillations between $|0\rangle$ 
and $| \pm 1\rangle$. According to Ref. [64], when $|\Delta| \gg u$, the effective Hamiltonian of this system reduces to the same framework of the $N$-qubit Dicke Hamiltonian in Eq. (1) as

$$
H_{\mathrm{eff}}=w_{b} b^{\dagger} b+\frac{u^{2}}{\Delta} G_{z}+\lambda_{\mathrm{eff}}\left(G_{+}+G_{-}\right)\left(b^{\dagger}+b\right)
$$

where $b$ is the oscillator operator of the fundamental oscillating mode with mechanical vibration frequency $w_{b} . G_{z}$ and $G_{ \pm}$are the collective angular-momentum operators for the bright state $|B\rangle=(1 / \sqrt{2})(|+1\rangle+|-1\rangle)$ and the dark state $|D\rangle=(1 / \sqrt{2})(|+1\rangle-|-1\rangle)$ in each NV center, where $G_{z}=\sum_{h=1}^{N}\left(|B\rangle_{h}\langle B|-| D\rangle_{h}\langle D|\right), G_{+}=\sum_{h=1}^{N}|B\rangle_{h}\langle D|$, and $G_{-}=$ $\sum_{h=1}^{N}|D\rangle_{h}\langle B|$. With current experiment parameters $[65,66]$ and suitable drivings, the nanotube vibrates at a frequency $w_{b} \approx 1 \mathrm{kHz}$ and the effective frequency $u^{2} / \Delta$ of NV center can be tuned to $10 \mathrm{MHz}$, i.e., the condition $\Omega / w_{0} \rightarrow \infty$ for finite $N$ playing the role of a thermodynamic limit is satisfied. At the same time, $|\Delta| \gg u$ is still valid, because $\Delta$ is compared with $u$, for example, $\Delta$ can be a quantity of $1 \mathrm{GHz}$ and $u$ can be a quantity of $10 \mathrm{MHz}$. The effective magnetomechanical coupling strength has a relation as $\lambda_{\text {eff }} \propto \frac{1}{d^{2}}$, which increases quickly just by decreasing $d$ to reach the critical point of QPT. When the strong-coupling regime is reached, i.e., $\lambda_{\text {eff }}$ exceeds both the electronic spin decay rate and the intrinsic damping rate of the mechanical mode, the dephasing effect on the QPT can be ignored reasonably.

\section{Conclusion}

In conclusion, we have studied the Dicke model beyond the thermodynamic limit. We combine the analytical methods of Schrieffer-Wolff transformation and BornOppenheimer approximation, and derive the eigenenergy and eigenstate of the normal and superradiant phases when the ratio of the qubit transition frequency to the oscillator frequency approaches infinity, demonstrating that the ground state undergoes a secondorder quantum phase transition. At the critical point, the effective qubit-oscillator coupling strength is quadratically enhanced by the number of qubits. We use the variational method to derive the leading-order correction for the finite-frequency ratio and show that the quartic correction term becomes a major contribution to the ground state energy when $N$ is large enough. Under the Kibble-Zurek mechanism, the universal scaling between the residual energy and the number of qubits is demonstrated to become an inverse-proportion relation. Finally, we analyze a promising experiment device to realize this $N$-qubit Dicke model. In the future, we hope to generalize the present method to other many-body systems, and find more novel QPTs beyond the thermodynamic limit, offering simple and applicable approaches for the experimental realization of QPT.

\section{Appendix A: Normal phase}

The free Hamiltonian $H_{0}$ has $N+1$ decoupled collective-qubit subspaces $\Gamma_{Q_{1}}=\{|j,-j\rangle\}$, $\Gamma_{Q_{2}}=\{|j,-j+1\rangle\}, \Gamma_{Q_{3}}=\{|j,-j+2\rangle\}, \ldots, \Gamma_{Q_{N+1}}=\{|j, j\rangle\}$. The decoupled collective-qubit subspaces of $H_{0}$ mean that there is no any interaction term between arbitrary two different subspaces. Here, we point at $H_{0}$ for decoupled subspaces, and do not point at the whole Hamiltionian $H$. There are different " $m$ " values, not " $j$ " values, that give the decoupled subspaces. When the ratio of the qubit transition frequency to the oscillator frequency 
approaches infinity, i.e., $\Omega / w_{0} \rightarrow \infty$, the eigenstate of $H_{0}$ with the lowest energy corresponds to that of the harmonic oscillator restricted in $\Gamma_{Q_{1}}$. A macroscopic quantum tunneling, under the adiabatic condition of $\Omega / w_{0} \rightarrow \infty$, for large $N$ the tunneling splitting vanishes as $\exp (-N)$, while for small $N$ the tunneling splitting disappears as $\sqrt{N} / \exp (N)$, as proved in $[67,68]$. Therefore, the ground state has no degeneracy and is unique.

To find a unitary transformation $U$ that decouples the mutual interaction between $\Gamma_{\mathrm{Q}_{1}}$ and $\Gamma_{Q_{2}}$ caused by the perturbation $V$, we apply Schrieffer-Wolff transformation to the Dicke Hamiltonian in the normal phase. Under the unitary transformation $U=e^{S}$, where the operator $S$ is anti-Hermitian and block-off-diagonal within $\Gamma_{Q_{1}}$, the system Hamiltonian becomes

$$
H^{\prime}=e^{-S} H e^{S}=\sum_{k=0}^{\infty} \frac{[H, S]^{(k)}}{k !},
$$

where $[H, S]^{(k)}=\left[[H, S]^{(k-1)}, S\right]$ with $[H, S]^{(0)}=H$. By dividing $H^{\prime}$ into the diagonal and offdiagonal parts and requiring that the off-diagonal part becomes zero up to fourth order in $\lambda$, we find the special anti-Hermitian operator $S$ with the approximate form

$$
\begin{aligned}
& S=\lambda S_{1}+\lambda^{3} S_{3}+O\left(w_{0}^{2} / \Omega^{2}\right), \\
& S_{1}=\frac{1}{\Omega}\left(a^{\dagger}+a\right)\left(J_{+}-J_{-}\right), \\
& S_{3}=-\frac{4}{3 \Omega^{3}}\left(a^{\dagger}+a\right)^{3}\left(J_{+}-J_{-}\right) .
\end{aligned}
$$

With this approximate $S$ and neglect the higher order terms $O\left(w_{0}^{2} / \Omega^{2}\right)$, the transformed Hamiltonian becomes

$$
\begin{aligned}
H^{\prime} \simeq & H_{0}+\frac{1}{2}\left[\left[H_{0}, \lambda S_{1}\right], \lambda S_{1}\right]-\left[V, \lambda S_{1}\right]+\frac{1}{2}\left[\left[H_{0}, \lambda^{3} S_{3}\right], \lambda S_{1}\right] \\
& +\frac{1}{2}\left[\left[H_{0}, \lambda S_{1}\right], \lambda^{3} S_{3}\right]+\frac{1}{24}\left[\left[\left[\left[H_{0}, \lambda S_{1}\right], \lambda S_{1}\right], \lambda S_{1}\right], \lambda S_{1}\right] \\
& -\left[V, \lambda^{3} S_{3}\right]-\frac{1}{6}\left[\left[\left[V, \lambda S_{1}\right], \lambda S_{1}\right], \lambda S_{1}\right] \\
= & w_{0} a^{\dagger} a+\Omega J_{z}+\frac{2 \lambda^{2}}{\Omega}\left(a^{\dagger}+a\right)^{2} J_{z}-\frac{w_{0} \lambda^{2}}{\Omega^{2}}\left(J_{+}-J_{-}\right)^{2} \\
& -\frac{2 \lambda^{4}}{\Omega^{3}}\left(a^{\dagger}+a\right)^{4} J_{z} .
\end{aligned}
$$

For infinite ratio $\Omega / w_{0} \rightarrow \infty$, we project $H^{\prime}$ into $\Gamma_{Q_{1}}$ and keep the terms to the second order of the qubit-oscillator coupling strength, we obtain an effective one-dimensional oscillator Hamiltonian in the normal phase

$$
\begin{aligned}
H_{n p} & =\left\langle\frac{N}{2},-\frac{N}{2}\left|H^{\prime}\right| \frac{N}{2},-\frac{N}{2}\right\rangle \\
& \simeq w_{0} a^{\dagger} a-\frac{N \lambda^{2}}{\Omega}\left(a^{\dagger}+a\right)^{2}-\frac{N \Omega}{2} .
\end{aligned}
$$


$H_{n p}$ in Eq. (a6) can be exactly diagonalized by applying a squeezing operator

$$
P(r)=e^{\frac{r}{2}\left(a^{\dagger 2}-a^{2}\right)},
$$

where $r$ is the squeezing parameter. This squeezing transformation leads to

$$
\begin{aligned}
H_{n p}^{\prime}= & P^{\dagger}(r) H_{n p} P(r) \\
= & {\left[w_{0} \cosh (2 r)-\frac{2 N \lambda^{2}}{\Omega} e^{2 r}\right] a^{\dagger} a } \\
& +\left[\frac{1}{2} w_{0} \sinh (2 r)-\frac{N \lambda^{2}}{\Omega} e^{2 r}\right]\left(a^{\dagger 2}+a^{2}\right) \\
& -\frac{N \Omega}{2}-\frac{N \lambda^{2}}{\Omega} e^{2 r}+w_{0} \sinh ^{2} r .
\end{aligned}
$$

With the choice of the squeezed parameter $r_{n p}=-\ln \left[1-4 N \lambda^{2} /\left(w_{0} \Omega\right)\right] / 4$, the coefficient of $\left(a^{\dagger 2}+a^{2}\right)$ term vanishes, so that $H_{n p}^{\prime}$ is diagonalized as

$$
H_{n p}^{\prime}=\epsilon_{n p} a^{\dagger} a+E_{n p}
$$

with the excitation energy

$$
\epsilon_{n p}=w_{0} \sqrt{1-\frac{4 N \lambda^{2}}{w_{0} \Omega}}
$$

and the ground-state energy

$$
E_{n p}=\frac{1}{2}\left(\epsilon_{n p}-w_{0}-N \Omega\right) .
$$

Crucially, the excitation energy $\epsilon_{n p}$ is real only when

$$
1-\frac{4 N \lambda^{2}}{w_{0} \Omega}>0
$$

or equivalently

$$
g=\frac{2 \sqrt{N} \lambda}{\sqrt{w_{0} \Omega}}<1
$$

Thus, the excitation energy $\epsilon_{n p}$ is a positive real value for $g<1$, corresponding to the normal phase. In this phase, the eigenstates and eigenenergies of $H_{n p}^{\prime}$ are

$$
\begin{aligned}
& \left|\phi_{n p}^{n}\right\rangle=P\left(r_{n p}\right)|n\rangle\left|\frac{N}{2},-\frac{N}{2}\right\rangle, \\
& E_{n p}^{n}=n \epsilon_{n p}+E_{n p} .
\end{aligned}
$$

Compared with the Rabi model [12], the qubit-oscillator coupling required for the quantum phase transition is reduced by a factor $\sqrt{N}$. 


\section{Appendix B: Superradiant phase}

When $g>1$, we see that the excitation energy $\epsilon_{n p}$ is imaginary, which implies that the number of photons in the oscillator becomes proportional to $\Omega /\left(w_{0} N\right)$ and acquires a macroscopic occupation, corresponding to the superradiant phase. In this phase, the higher-order terms in $O\left(w_{0} N / \Omega\right)$ can not be neglected, meaning that the assumption of low-energy subspace restricted in $\Gamma_{Q_{1}}$ is invalid. To capture the physics of superradiant phase, we displace the oscillator in $H$ by applying the displacement operator $D(\alpha)=e^{\alpha\left(a^{\dagger}-a\right)}$ ( $\alpha$ is a displacement parameter), which corresponds to the displacement transformation

$$
a^{\dagger} \rightarrow a^{\dagger}+\alpha
$$

Then, the original Hamiltonian is transformed to

$$
\begin{aligned}
H_{s p}= & D^{\dagger}(\alpha) H D(\alpha) \\
= & w_{0} a^{\dagger} a+\left[w_{0} \alpha-\lambda\left(J_{+}+J_{-}\right)\right]\left(a^{\dagger}+a\right)+\Omega J_{z} \\
& -2 \lambda \alpha\left(J_{+}+J_{-}\right)+w_{0} \alpha^{2} .
\end{aligned}
$$

The main obstacle of dealing with $H_{s p}$ is that it is hard to give the complete eigenstates of the qubit part $\Omega J_{z}-2 \lambda \alpha\left(J_{+}+J_{-}\right)$due to the high-dimensional complexity of collective-qubit subspaces when $N$ increases. To overcome this obstacle, we here consider the effective potential of the oscillator by means of Born-Oppenheimer approximation [32], instead of the matrix diagonalization method used in Rabi model [12]. For $\Omega / w_{0} \rightarrow \infty$, we can say that the qubits remain in the lowest energy eigenstate $|N / \widetilde{2,-N} / 2\rangle$ and this state changes adiabatically following the dynamics of the slow oscillator [32]. Since the coupling between the qubits and oscillator is mediated by the oscillator's position operator $x$, we start the calculation by finding the qubits' lowest eigenenergy for the slow oscillator. Assume that $x$ has a well-defined value, the qubit part of $H_{s p}$ can be rewritten as

$$
H_{q}(x)=\Omega J_{z}-\left(2 \lambda \alpha+\sqrt{2 w_{0}} \lambda x\right)\left(J_{+}+J_{-}\right)
$$

The lowest eigenenergy of this Hamiltonian in $\Gamma_{Q}$ is given by

$$
K_{g}(x)=-N \sqrt{\frac{\Omega^{2}}{4}+\left(2 \lambda \alpha+\sqrt{2 w_{0}} \lambda x\right)^{2}} .
$$

When the qubits remain in $|N / \widetilde{2,-N} / 2\rangle$, the oscillator's effective potential acquires a new contribution from the qubits as

$$
\begin{aligned}
V_{\mathrm{eff}}(x)= & \frac{1}{2} w_{0}^{2} x^{2}+\sqrt{2 w_{0}} w_{0} \alpha x \\
& -N \sqrt{\frac{\Omega^{2}}{4}+\left(2 \lambda \alpha+\sqrt{2 w_{0}} \lambda x\right)^{2}} .
\end{aligned}
$$

Note that the position variable $x$ appears inside the square root of $K_{g}$ and the effective potential in Eq. (b5), corresponding to a nonlinear harmonic potential. Therefore, it is impossible to obtain general analytical solutions and we consider the specially approximate solution in the following. 
In the limit $\Omega \gg \lambda|x|$, the effective potential in Eq. (b5) can be approximated as

$$
\begin{aligned}
V_{\mathrm{eff}}(x) \simeq & \frac{1}{2} w_{0}^{2} x^{2}+\sqrt{2 w_{0}} w_{0} \alpha x \\
& -\frac{N}{2} \sqrt{\Omega^{2}+16 \lambda^{2} \alpha}\left[1+\frac{8 \sqrt{2} \lambda^{2} \alpha x+4 w_{0} \lambda^{2} x^{2}}{\Omega^{2}+16 \lambda^{2} \alpha^{2}}\right. \\
& \left.-\frac{1}{8}\left(\frac{16 \sqrt{2} \lambda^{2} \alpha x+8 w_{0} \lambda^{2} x^{2}}{\Omega^{2}+16 \lambda^{2} \alpha^{2}}\right)^{2}\right] \\
\simeq & \frac{1}{2} \epsilon_{s p}^{2}\left(x+\frac{\sqrt{2 w_{0}} w_{0} \alpha-\frac{4 \sqrt{2} N \lambda^{2} \alpha}{\tilde{\Omega}}}{\epsilon_{s p}^{2}}\right)^{2}+V_{\min },
\end{aligned}
$$

where

$$
\begin{aligned}
& \tilde{\Omega}=\sqrt{\Omega^{2}+16 \lambda^{2} \alpha^{2}}, \\
& \epsilon_{s p}^{2}=w_{0}^{2}-\frac{4 N w_{0} \lambda^{2}}{\tilde{\Omega}}+\frac{64 N \lambda^{4} \alpha^{2}}{\tilde{\Omega}^{3}}, \\
& V_{\min }=-\frac{N \tilde{\Omega}}{2}-\frac{\left(\sqrt{2 w_{0}} w_{0} \alpha-\frac{4 \sqrt{2} N \lambda^{2} \alpha}{\tilde{\Omega}}\right)^{2}}{2 \epsilon_{s p}^{2}} .
\end{aligned}
$$

The results show that the oscillator's effective potential is changed due to coupling to the qubits. On one hand, the equilibrium position of the oscillator is moved by a quantity proportional to $N \alpha / \tilde{\Omega}$. On the other hand, the oscillator's effective frequency $\epsilon_{s p}$ is reduced by a quantity roughly proportional to $N / \tilde{\Omega}$. The minimum potential can be obtained by setting $d V_{\min } / d \alpha=0$, where the solution of $\alpha$ is derived as

$$
\alpha_{g}= \pm \sqrt{\frac{N \Omega}{4 w_{0} g^{2}}\left(g^{4}-1\right)} .
$$

With these two independent choices of $\alpha_{g}$, the oscillator's effective frequency becomes

$$
\epsilon_{s p}=w_{0} \sqrt{1-g^{-4}}
$$

which is real for $g>1$, and the corresponding ground-state energy is

$$
E_{s p}=\frac{1}{2}\left(\epsilon_{s p}-w_{0}\right)-\frac{N \Omega}{4}\left(g^{2}+g^{-2}\right) .
$$

Note that the expression of the ground state $|N / \widetilde{2,-N} / 2\rangle$ can be found in Ref. [32] within the spin space.

\section{Appendix C: Quantum phase transition}

The behavior of the system's ground state is characterized by the excitation energies $\epsilon_{n p}$ and $\epsilon_{s p}$ of the effective oscillator when the qubits are in the lowest energy eigenstate. These excitation energies as a function of the coupling strength for different number of qubits are displayed in Fig. 3. The result shows that as the coupling strength approaches the critical 

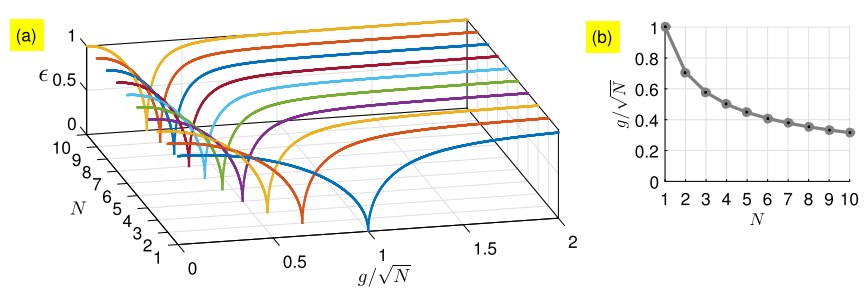

Figure 3 (a) Excitation energy $\epsilon$ of the Dicke Hamiltonian as a function of the coupling strength for different number of qubits in the limit $\Omega / w_{0} \gg 1$. (b) Coupling strength versus $N$ at the critical point. We set $\Omega=100 w_{0}$. The vanishing of $\epsilon$ at the critical point $g=1$ indicates the appearance of the QPT
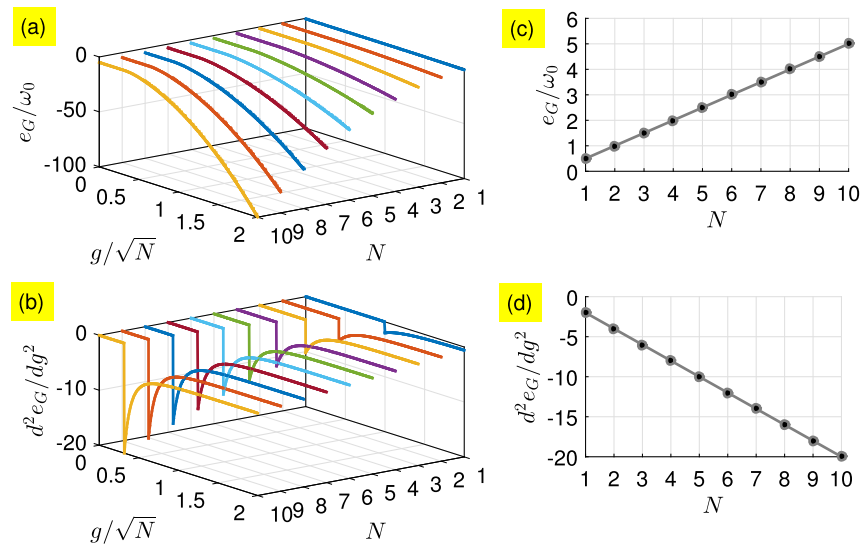

Figure 4 (a) The rescaled ground-state energy $e_{G}$ and (b) its second derivative $d^{2} e_{G} / d g^{2}$ as a function of the coupling strength for different number of qubits in the limit $\Omega / w_{0} \gg 1$. (c) $e_{G}$ and (d) $d^{2} e_{G} / d g^{2}$ versus $N$ at the critical point. We set $\Omega=100 w_{0}$

point $g=1$, the excitation energy of the oscillator vanishes as $\epsilon(g) \propto|g-1|^{z v}$ where $z v=$ $1 / 2$ is defined as the dynamical critical exponent, indicating the appearance of the QPT. As shown in Eq. (a13), the qubit-oscillator coupling strength for generating QPT can be quadratically reduced with the increasing of the number of qubits. This is due to the fact that the effective qubit-oscillator coupling is enhanced by the collective interaction of the qubits.

The rescaled ground-state energy $e_{G}=\left(w_{0} / \Omega\right) E$ is $-N w_{0} / 2$ for $g<1$ and $-N w_{0}\left(g^{2}+g^{-2}\right) / 4$ for $g>1$, which is a continuous curve for different number of qubits shown in Fig. 4(a), but the second derivative $d^{2} e_{G} / d g^{2}$ in Fig. 4(b) exhibits a discontinuity for different number of qubits at the critical point $g=1$, clearly revealing the second-order nature of this QPT. $e_{G}$ in Fig. 4(a) tends towards a value of $-\mathrm{Nw}_{0} / 2$ when $g \rightarrow 1$ from either direction, which linearly depends on the number of qubits, and becomes infinite in the asymptotic limit of $g \rightarrow \infty$. As shown in Fig. 4(b), $d^{2} e_{G} / d g^{2}$ vanishes as $g \rightarrow 1$ and tends towards a constant of $-N w_{0} / 2$ in the limit of $g \rightarrow \infty$. The physics behind this QPT is that the infinite ratio $\Omega / w_{0} \rightarrow \infty$ for finite $N$ plays the role of a thermodynamic limit, which allows the spectral gap to vanish at the critical point. 
Figure 5 The corrections due to finite frequency ratio in (a) $e_{G}$ (b) $n_{C,}$ (c) $\Delta x$, and (d) $\Delta p$ as a function of the number of qubits. We set $\Omega=1000 w_{0}$
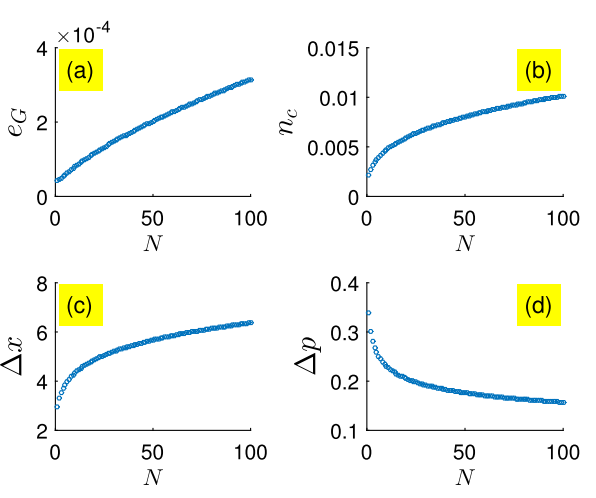

\section{Appendix D: Corrections due to finite frequency ratio}

For finite ratios of $\Omega / w_{0} \gg 1$, we derive an analytical leading-order correction by using the variational method [12]. By keeping $H^{\prime}$ up to the fourth order term in $\lambda / \Omega$ and projecting it into $\Gamma_{\mathrm{Q}_{1}}$, we obtain

$$
H_{n p}^{\Omega}=H_{n p}+\frac{w_{0}^{2} g^{4}}{16 N \Omega}\left(a^{\dagger}+a\right)^{4}+\frac{w_{0}^{2} g^{2}}{4 \Omega},
$$

which shows that the leading-order correction to $H_{n p}$ corresponds to a quartic potential for the oscillator, with a strength coefficient inversely proportional to the number of qubits. We assume the ground state of $H_{n p}^{\Omega}$ to be the squeezed state $\left|\psi_{0}(s)\right\rangle=P(s)|0\rangle$ with a variational parameter $s$, which provides the corresponding variational energy

$$
\begin{aligned}
E_{0}(s)= & \left\langle\psi_{0}(s)\left|H_{n p}^{\Omega}\right| \psi_{0}(s)\right\rangle \\
= & \frac{w_{0}}{2} \cosh (2 s)-\frac{w_{0} g^{2}}{4} e^{2 s}+\frac{3 w_{0}^{2} g^{4}}{16 N \Omega} e^{4 s}-\frac{N}{2} \Omega \\
& +\frac{w_{0}^{2} g^{2}}{4 \Omega}-\frac{w_{0}}{2} .
\end{aligned}
$$

Through $\partial E_{0}(s) / \partial s=0$, we have

$$
\frac{3 w_{0} g^{4}}{2 N \Omega} e^{6 s}+\left(1-g^{2}\right) e^{4 s}-1=0
$$

$\partial^{2} E_{0}(s) / \partial s^{2}>0$ is valid for any real $s$, demonstrating that the ground state $\left|\psi_{0}(s)\right\rangle$ has the solution of $s$ in Eq. (d3). At the critical point $g=1$, the solution of $s$ has a particularly simple form

$$
s_{\min }=\frac{1}{6} \ln \left(\frac{2 N \Omega}{3 w_{0}}\right) .
$$

With this variational solution at critical point, we find the corrections for the rescaled ground state energy

$$
\begin{aligned}
e_{G} & =\frac{w_{0}}{\Omega}\left[\left\langle\psi_{0}\left(s_{\min }\right)\left|H_{n p}^{\Omega}\right| \psi_{0}\left(s_{\min }\right)\right\rangle-E_{n p}\right] \\
& \simeq \frac{w_{0}}{8}(2+N)\left(\frac{2 N}{3}\right)^{-1 / 3}\left(\frac{\Omega}{w_{0}}\right)^{-4 / 3}
\end{aligned}
$$




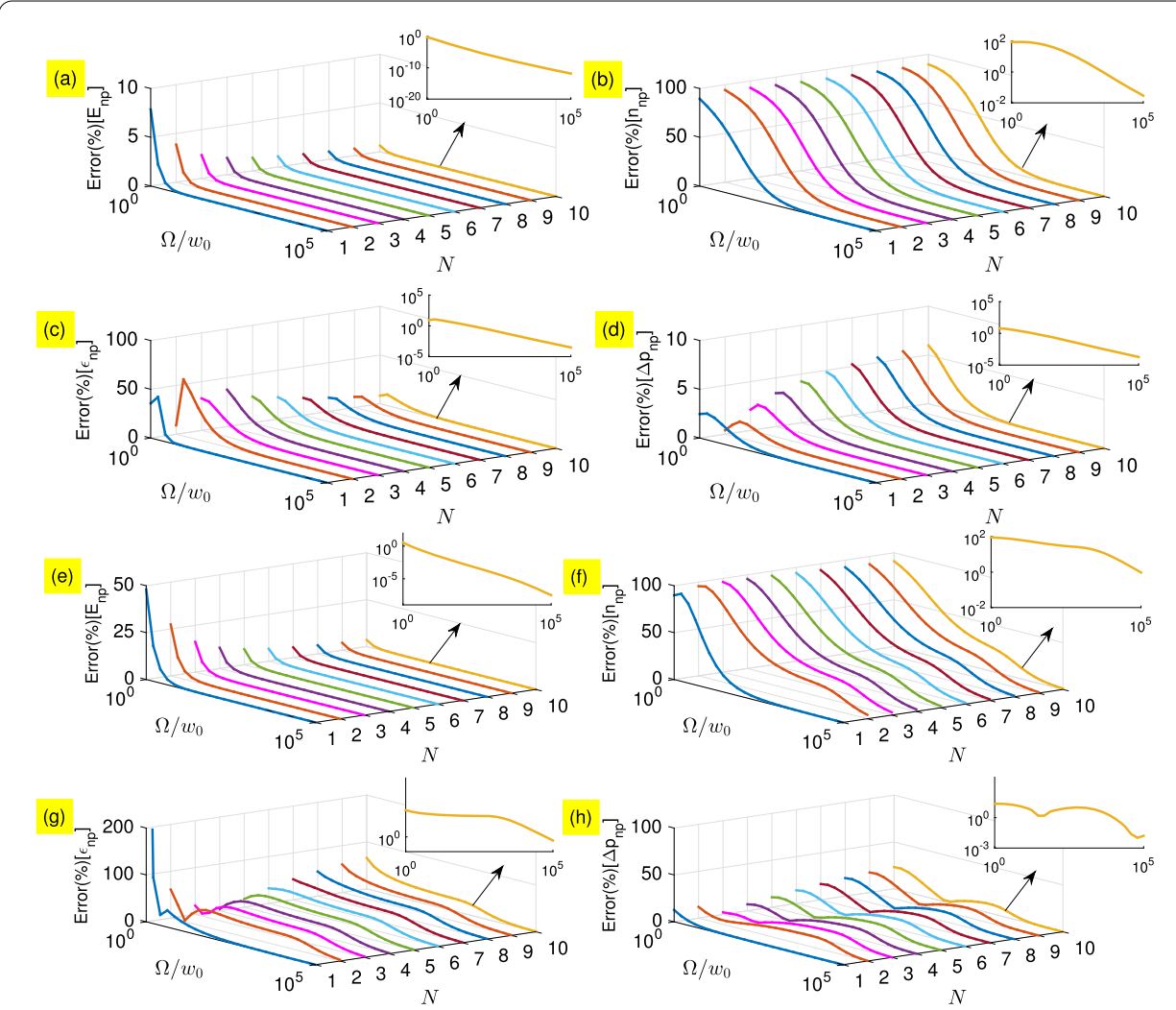

Figure 6 Errors of solutions from the effective Hamiltonian of Eq. (a5) with the numerical solutions at $g=\frac{1}{2}$ $[(\mathbf{a})-(\mathbf{d})]$ and $g=1[(\mathbf{e})-(\mathbf{h})]$. At the top-right corner of each subgraph, the detail between the error and $\Omega / w_{0}$ is magnified when $N=10$

for the rescaled photon number $n_{c}$

$$
\begin{aligned}
n_{c} & =\frac{w_{0}}{\Omega}\left\langle\psi_{0}\left(s_{\min }\right)\left|a^{\dagger} a\right| \psi_{0}\left(s_{\min }\right)\right\rangle \\
& \simeq \frac{1}{6} N^{1 / 3}\left(\frac{2 \Omega}{3 w_{0}}\right)^{-2 / 3},
\end{aligned}
$$

and for the variance of quadratures $\Delta x, \Delta p$

$$
\begin{aligned}
& \Delta x=e^{s_{\min }}=N^{1 / 6}\left(\frac{2 \Omega}{3 w_{0}}\right)^{1 / 6}, \\
& \Delta p=e^{-s_{\min }}=N^{-1 / 6}\left(\frac{2 \Omega}{3 w_{0}}\right)^{-1 / 6} .
\end{aligned}
$$

For the excitation energy at the critical point, the correction is given by

$$
\begin{aligned}
\epsilon_{s}= & \left\langle 1\left|P^{\dagger}\left(s_{\min }\right) H_{n p}^{\Omega} P\left(s_{\min }\right)\right| 1\right\rangle-\langle 0| P^{\dagger}\left(s_{\min }\right) H_{n p}^{\Omega} \\
& \otimes P\left(s_{\min }\right)|0\rangle \\
= & w_{0} N^{-1 / 3}\left(\frac{2 \Omega}{3 w_{0}}\right)^{-1 / 3} .
\end{aligned}
$$



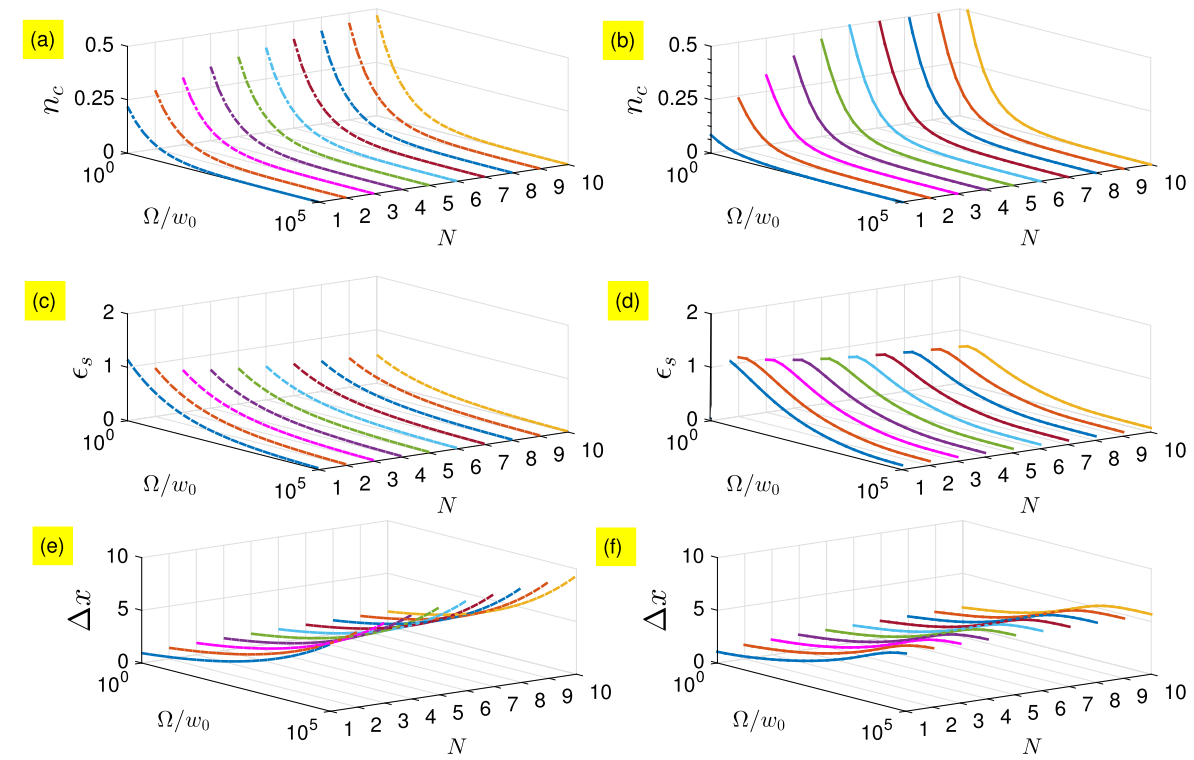

(g)
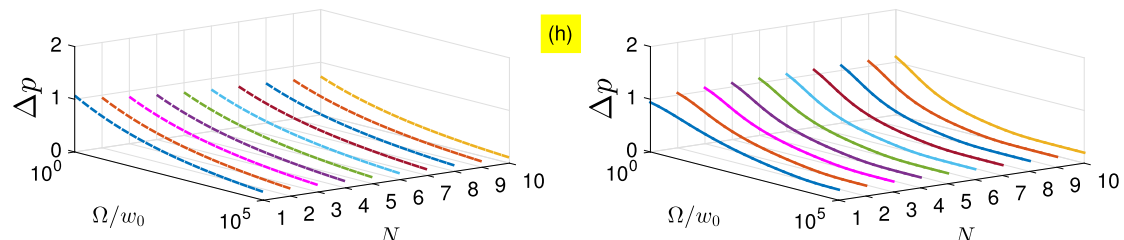

Figure 7 Comparison of solutions from the leading order terms [Eqs. (d5)-(d8)] with the corresponding numerical solutions at the critical point $g=1: n_{c}[(\mathbf{a})-(\mathbf{b})], \epsilon_{s}[(\mathbf{c})-(\mathbf{d})], \Delta x[(\mathbf{e})-(\mathbf{f})]$, and $\Delta p[(\mathbf{g})-(\mathbf{h})]$. The dashed lines plot their leading order terms with analytical solutions, and the solid lines plot the corresponding numerical solutions

The above results indicate that the correction term $\left(a^{\dagger}+a\right)^{4}$ becomes a major contribution to the ground state energy at the critical point when $N$ is large enough, as shown in Fig. 5.

\section{Appendix E: Justified estimate for the analytical result}

To check the validity of the above analytical results, we perform numerical proofs in this section. In Fig. 6, we compare errors between the analytical solution of Eq. (a5) and the numerically exact solution when $N$ is finite. From the result of Fig. 6, the deviation between the analytical and numerical solutions is very small and decays fast as $\Omega / w_{0}$ increases, indicating that $H^{\prime}$ is an excellent approximation of the Dicke model for $\Omega / w_{0} \gg 1$ and finite $N$.

As shown in Fig. 7, we make comparisons of the leading order terms between the analytical and numerical solutions when $N$ is finite. By keeping the higher order corrections, the difference between the analytical prediction and exact result is very small. However, it is not necessary to expect our approximation results to be exactly accurate for small values of $\Omega / w_{0}$ and finite $N$. We are only interested in scaling behaviors for large values of $\Omega / w_{0}$ and finite $N$, and our analytical approximations correctly predict them. Therefore, it is safe to predict quench dynamics of the $N$-qubit Dicke model based on the above analytical approximations in the following section. 


\section{Acknowledgements}

LTS thanks Prof. Pengbo Li for valuable discussions.

\section{Funding}

The National Natural Science Foundation of China (Grant Nos. 11674060, 11774058, and 11705030), the Natural Science Foundation of Fujian Province (Grant Nos. 2017J05005 and 2016J01018), and the Fujian Provincial Department of Education under Grant No. JZ160422.

\section{Availability of data and materials}

The data sets used or analysed during the current study are available from the corresponding author on reasonable request.

\section{Competing interests}

The authors declare that they have no competing interests.

\section{Authors' contributions}

ZCS contributed the detailed analysis of the physics behind the quantum phase transition. YZB plotted all the figures. HZW wrote the matlab codes. ZRZ derived Eqs. (d1)-(d9). SBZ gave the main idea of this model. All authors read and approved the final manuscript.

\section{Publisher's Note}

Springer Nature remains neutral with regard to jurisdictional claims in published maps and institutional affiliations.

Received: 10 November 2019 Accepted: 23 December 2019 Published online: 06 January 2020

\section{References}

1. Dicke RH. Coherence in spontaneous radiation processes. Phys Rev. 1954;93:99.

2. Brandes T. Coherent and collective quantum optical effects in mesoscopic systems. Phys Rep. 2005;408:315

3. Hepp K, Lieb E. On the superradiant phase transition for molecules in a quantized radiation field: the Dicke maser model. Ann Phys. 1973;76:360.

4. Baumann K, Guerlin C, Brennecke F, Esslinger T. Dicke quantum phase transition with a superfluid gas in an optical cavity. Nature (London). 2010;464:1301.

5. Bloch I, Dalibard J, Nascimbène S. Quantum simulations with ultracold quantum gases. Nat Phys. 2012;8:267.

6. Nagy D, Domokos P. Critical exponent of quantum phase transitions driven by colored noise. Phys Rev A. 2016;94:063862

7. Dimer F, Estienne B, Parkins A, Carmichael H. Proposed realization of the Dicke-model quantum phase transition in an optical cavity QED system. Phys Rev A. 2007;75:013804.

8. Kim K, Chang MS, Korenblit S, Islam R, Edwards EE, Freericks JK, Lin GD, Duan LM, Monroe C. Quantum simulation of frustrated Ising spins with trapped ions. Nature. 2010;465:590.

9. Bermudez A, Plenio MB. Spin peierls quantum phase transitions in Coulomb crystals. Phys Rev Lett. 2012;109:010501.

10. Polkovnikov A, Sengupta K, Silva A, Vengalattore M. Colloquium: nonequilibrium dynamics of closed interacting quantum systems. Rev Mod Phys. 2011;83:863

11. Eisert J, Friesdorf M, Gogolin C. Quantum many-body systems out of equilibrium. Nat Phys. 2015:11:124

12. Hwang MJ, Puebla R, Plenio MB. Quantum phase transition and universal dynamics in the Rabi model. Phys Rev Lett. 2015:115:180404.

13. Schiró M, Joshi C, Bordyuh M, Fazio R, Keeling J, Türeci HE. Exotic attractors of the nonequilibrium Rabi-Hubbard model. Phys Rev Lett. 2016;116:143603.

14. Abdumalikov AA Jr, Astafiev O, Nakamura Y, Pashkin YA, Tsai JS. Vacuum Rabi splitting due to strong coupling of a flux qubit and a coplanar-waveguide resonator. Phys Rev B. 2008;78:180502(R).

15. Forn-Díaz P, Lisenfeld J, Marcos D, García-Ripoll JJ, Solano E, Harmans CJPM, Mooij JE. Observation of the Bloch-Siegert shift in a qubit-oscillator system in the ultrastrong coupling regime. Phys Rev Lett. 2010;105:237001.

16. Niemczyk T, Deppe F, Huebl H et al. Circuit quantum electrodynamics in the ultrastrong-coupling regime. Nat Phys. 2010;6:772.

17. Günter G, Anappara AA, Hees J et al. Sub-cycle switch-on of ultrastrong light-matter interaction. Nature (London). 2009;458:178.

18. Todorov Y, Andrews AM, Colombelli R, Liberato SD, Ciuti C, Klang P, Strasser G, Sirtori C. Ultrastrong light-matter coupling regime with polariton dots. Phys Rev Lett. 2010;105:196402.

19. Scalari G, Maissen C, Turčinková D et al. Ultrastrong coupling of the cyclotron transition of a $2 \mathrm{D}$ electron gas to a THz metamaterial. Science. 2012;335:1323.

20. Crespi A, Longhi S, Osellame R. Photonic realization of the quantum Rabi model. Phys Rev Lett. 2012;108:163601.

21. Zheng SB. Dicke-like quantum phase transition and vacuum entanglement with two coupled atomic ensembles. Phys Rev A. 2011;84:033817.

22. Shen LT, Yang ZB, Wu HZ, Zheng SB. Ground state of an ultrastrongly coupled qubit-oscillator system with broken inversion symmetry. Phys Rev A. 2016;93:063837.

23. Viehmann O, von Delft J, Marquardt F. Superradiant phase transitions and the standard description of circuit QED. Phys Rev Lett. 2011;107:113602.

24. Jaako T, Xiang ZL, Garcia-Ripoll JJ, Rabl P. Ultrastrong-coupling phenomena beyond the Dicke model. Phys Rev A 2016:94:033850

25. Emary C, Brandes T. Quantum chaos triggered by precursors of a quantum phase transition: the Dicke model. Phys Rev Lett. 2003:90:044101.

26. Emary C, Brandes T. Chaos and the quantum phase transition in the Dicke model. Phys Rev E. 2003;67:066203. 
27. Bastidas VM, Emary C, Regler B, Brandes T. Nonequilibrium quantum phase transitions in the Dicke model. Phys Rev Lett. 2012;108:043003.

28. Seiringer R. The excitation spectrum for weakly interacting bosons. Commun Math Phys. 2011;306:565.

29. Zurek WH, Dorner U, Zoller P. Dynamics of a quantum phase transition. Phys Rev Lett. 2005;95:105701.

30. Zhu SL, Wang Z, Zanardi P. Geometric quantum computation and multiqubit entanglement with superconducting qubits inside a cavity. Phys Rev Lett. 2005:94:100502.

31. Braak D. Solution of the Dicke model for N = 3. J Phys B, At Mol Opt Phys. 2013;46:224007.

32. Liberti G, Plastina F, Piperno F. Scaling behavior of the adiabatic Dicke model. Phys Rev A. 2006;74:022324.

33. Vidal J, Dusuel S. Finite-size scaling exponents in the Dicke model. Europhys Lett. 2006;74:817.

34. Tsyplyatyev O, Loss D. Dicke model: entanglement as a finite size effect. J Phys Conf Ser. 2009;193:012134.

35. Relaño A, Bastarrachea-Magnani MA, Lerma-Hernández S. Approximated integrability of the Dicke model. Europhys Lett. 2016;1 16:50005.

36. Bao L, Pan F, Li XT, Liang X, Draayer JP. The progressive solutions for the Dicke Hamiltonian. Eur Phys J Plus. 2015;130:126.

37. He S, Duan LW, Chen QH. Exact solvability, non-integrability, and genuine multipartite entanglement dynamics of the Dicke model. New J Phys. 2015;17:043033.

38. Lambert N, Emary C, Brandes T. Entanglement and the phase transition in single-mode superradiance. Phys Rev Lett. 2004;92:073602

39. Casteels W, Fazio R, Ciuti C. Critical dynamical properties of a first-order dissipative phase transition. Phys Rev A. 2017; $95: 012128$

40. Fink JM, Dombi A, Vukics A, Wallraff A, Domokos P. Observation of the photon-blockade breakdown phase transition. Phys Rev X. 2017;7:011012.

41. Xie QT, Cui S, Cao JP, Amico L, Fan H. Anisotropic Rabi model. Phys Rev X. 2014;4:021046.

42. Hwang MJ, Plenio MB. Quantum phase transition in the finite Jaynes-Cummings lattice systems. Phys Rev Lett. 2016;117:123602

43. Raimond JM, Brune M, Haroche S. Manipulating quantum entanglement with atoms and photons in a cavity. Rev Mod Phys. 2001;73:565.

44. Jaynes ET, Cummings FW. Comparison of quantum and semiclassical radiation theories with application to the beam maser. Proc IEEE. 1963;51:89.

45. Zheng SB, Guo GC. Efficient scheme for two-atom entanglement and quantum information processing in cavity QED. Phys Rev Lett. 2000;85:2392.

46. Irish EK. Generalized rotating-wave approximation for arbitrarily large coupling. Phys Rev Lett. 2007;99:173601.

47. Wu CF, Guo C, Wang Y, Wang GC, Feng XL, Chen JL. Generation of Dicke states in the ultrastrong-coupling regime of circuit QED systems. Phys Rev A. 2017;95:013845.

48. Chen QH, Wang C, He S, Liu T, Wang KL. Exact solvability of the quantum Rabi model using Bogoliubov operators, Phys Rev A. 2012;86:023822.

49. Zhong HH, Xie QT, Batchelor MT, Lee CH. Analytical eigenstates for the quantum Rabi model. J Phys A. 2013;46:415302

50. Du LH, Zhou XF, Zhou ZW, Zhou X, Guo GC. Generalized Rabi model in quantum-information processing including the $A^{2}$ term. Phys Rev A. 2012;86:014303.

51. Altintas F, Eryigit R. Dissipative dynamics of quantum correlations in the strong-coupling regime. Phys Rev A. 2013:87:022124

52. Braak D. Integrability of the Rabi model. Phys Rev Lett. 2011;107:100401.

53. Joshi C, Larson J, Spiller TP. Quantum state engineering in hybrid open quantum systems. Phys Rev A. 2016;93:043818.

54. Zheng H. Dynamics of a two-level system coupled to Ohmic bath: a perturbation approach. Eur Phys J B. 2004;38:559.

55. Solano E, Agarwal GS, Walther H. Strong-driving-assisted multipartite entanglement in cavity QED. Phys Rev Lett. 2003:90:027903.

56. Rostovtsev YV, Eleuch H, Svidzinsky A, Li H, Sautenkov V, Scully MO. Excitation of atomic coherence using off-resonant strong laser pulses. Phys Rev A. 2009;79:063833.

57. Cao X, You JQ, Zheng H, Nori F. A qubit strongly coupled to a resonant cavity: asymmetry of the spontaneous emission spectrum beyond the rotating wave approximation. New J Phys. 2011:13:073002

58. Ridolfo A, Leib M, Savasta S, Hartmann MJ. Photon blockade in the ultrastrong coupling regime. Phys Rev Lett. 2012;109:193602.

59. Ashhab S. Superradiance transition in a system with a single qubit and a single oscillator. Phys Rev A. 2013;87:013826.

60. Garziano L, Ridolfo A, De Liberato $S$ et al. Cavity QED in the ultrastrong coupling regime: photon bunching from the emission of individual dressed qubits. ACS Photonics. 2017:4:2345.

61. Kibble TWB. Topology of cosmic domains and strings. J Phys A. 1976;9:1387.

62. Zurek WH. Cosmological experiments in superfluid helium? Nature (London). 1985;317:505.

63. De Grandi C, Polkovnikov A. Quantum quenching, annealing and computation. Heidelberg: Springer; 2010.

64. Li PB, Xiang ZL, Rabl P, Nori F. Hybrid quantum device with nitrogen-vacancy centers in diamond coupled to carbon nanotubes. Phys Rev Lett. 2016;117:015502

65. Sapmaz S, Blanter YaM, Gurevich L, van der Zant HSJ. Carbon nanotubes as nanoelectromechanical systems. Phys Rev B. 2003;67:235414.

66. Aykol M, Hou B, Dhall R, Chang SW, Branham W, Qiu J, Cronin SB. Clamping instability and van der Waals forces in carbon nanotube mechanical resonators. Nano Lett. 2014:14:2426.

67. Chen G, Chen ZD, Liang JQ. Quantum tunneling in the adiabatic Dicke model. Phys Rev A. 2007;76:045801

68. Razavy M. Quantum theory of tunnelling. Singapore: World Scientific; 2003. 